\title{
The Preliminary Exploration about the Design of the Top Interface of the Gymnasium of Ice Sport based on the Dynamic Optimization of Natural Light Environment
}

\author{
Ying Liu ${ }^{1, a}$, Ge Yu ${ }^{1, b}$ and Changhe Wang ${ }^{1, c}$ \\ ${ }^{1}$ School of Architecture, Harbin Institute of Technology, Harbin, 150001, China \\ a niuniu12345000@163.com, ${ }^{\text {b }}$ yuge_hit@yeah.net, ${ }^{c}$ 1072202731@qq.com
}

Keywords: Gymnasium of ice sport, Top interface, Natural light environment, Dynamic optimization.

\begin{abstract}
In this paper, from the dynamic optimization perspective of the natural light environment, applying the value analysis to the top interface design of the gymnasium of ice sport, to explore of the design object of the top interface of the gymnasium of ice sport on the natural light environment. To break the design errors of quantitative indicators in the traditional light environment, and explain the dynamic characteristics of natural light in the competition hall. Through the analysis of the intrinsic link between ice sports hall function and the different user demand, to search the specific content of the top interface design of the gymnasium of ice sport, and put forward the dynamic optimization strategy of the gymnasium of ice sport top interface design.
\end{abstract}

\section{The Gymnasiums of Ice Sport Top Interface Design}

With the continuous improvement of the ice sports level and the advance of social sports resources sharing idea in our country, more and more gymnasiums of ice sport become public sports venues after big events, going towards the road of ice sports for popular service. Among them, to improve the public service, the contradiction between the reducing of the cost of public service and the cost of the maintenance and operation is the primary problems that troubled Chinese most of the gymnasiums of ice sport operators. It is necessary to point out that the gymnasiums of ice sport category not only including the gymnasiums which can hold figure skating and the other ice sports, but also including the special ice sports architectures having the characteristics of cold region, which including speed skating museum, hockey hall, curling hall ${ }^{[1]}$.

Top interface is one of three interfaces defining the space, the trend of gymnasium of ice sport top interface, the natural lighting forms, and the visual composition relationship between structural members with natural lighting, artificial lighting, air conditioning, and sound control equipment determine the overall image of the building interior space. Its design, construction and operation and maintenance all need to ensure a certain economic health, while the acquisition of economic health are inextricably linked to the effective use of the available resources. The gymnasium of ice sport top interface design long-term exists the practical problems including the blind copying sports architectural design results in other thermal areas, ignoring ice movement characteristics and the constrains of geographical, economic and climatic characteristics, causing the decline in sport comfort, cannot meet the function demand of user, operating costs continue to rise.

\section{The Relating Study on the Top Interface of the Gymnasiums of Ice Sport Top Interface Design}

In the cold area of the western developed countries, the building top interface energy consumption problem in the using process has already been subjected to the attention of the government and the masses, selecting the strain characteristics appropriate technology for the day lighting design of the regional climate characteristics. At present, the research of the construction design of the cold regions in China, multiply point at the roof structure and the vertical retaining structure, and so on.

In the design of the top interface of ice sport in our country, due to the lack of understanding of regional climate characteristics, the optimization problem of the natural light environment from the 
perspective of the regional climate is not solved well. The multiple existing designs copy of the existing buildings energy-saving mode in developed countries and a lot of technical means are blindly applied. This design method not only has no strain on the climate, making good thermal insulation, drainage, waterproof, anti snow design, and even brings greater energy consumption.

Through the field research on the gymnasiums of ice sport in the severe cold religions and the cold religions including Beijing, Shenyang, Changchun, Harbin and so on(Figure 1), founding the following several points problems existing in the top interface design of the gymnasiums of ice sport in our country such as: to emphasize sports hall symbol attribute, the roof shape design has become the focus, ignoring the organization and design of the competition hall top interface; the top interface sunroof open only consider the external shape, not in-depth analysis the relationship with the competition site and the impact on natural light environment. The day training of the gymnasium mostly make use of the artificial lighting, and the natural lighting cannot meet the needs of the daily training and exercise.

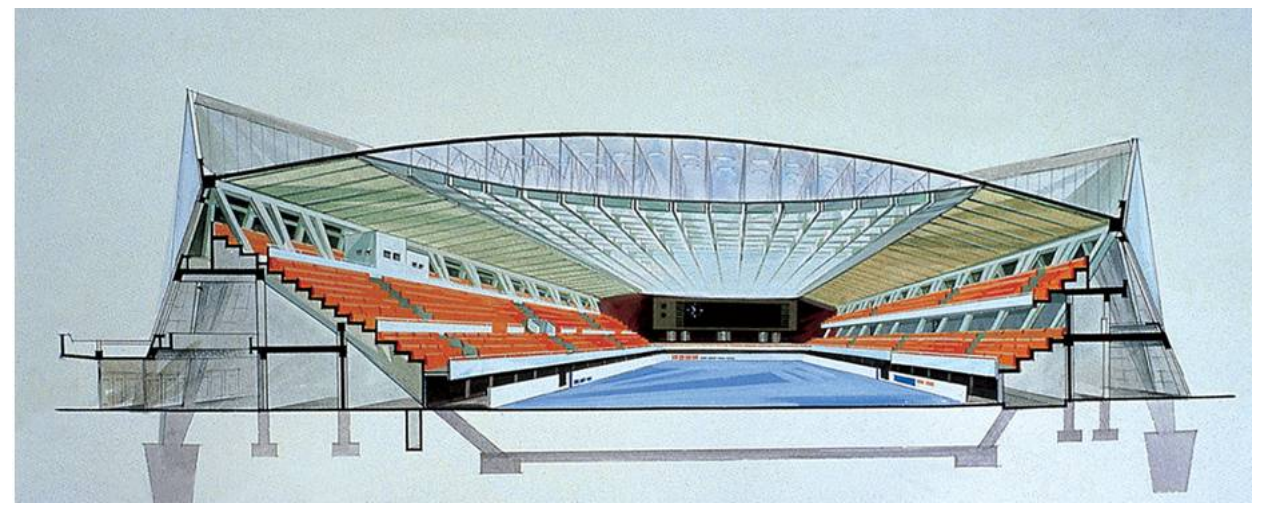

Fig.1 Cross-sectional view of Jilin hockey hall

\section{The Goal of the Top Interface Design of the Ice Sports Hall of the Gymnasiums of Ice Sport Top Interface Design}

Establishing the Dynamic Model of the Top Interface. Breaking through the traditional design concept, taking the dynamic model of the interface instead of the steady state model of the top interface. To fully meet the premise to satisfy the needs of users, comprehensive utilizing of appropriate technology to take the natural light into the stadium, improving the match hall space environment quality, meeting the day sports training and exercise, create ecological suitable, healthy and high quality the gymnasium of ice sport top interface and natural light environment of the competition hall.

Getting the "best" solution. Based on value analysis to study the gymnasiums of ice sport top interface function and the cost of reasonable matching, emphasizing the organic combination of the life-cycle function improved and reducing the cost, so as to obtain the "optimal" solutions of the gymnasiums of ice sport top interface dynamic optimization, to promote the realization of the overall value of the gymnasiums of ice sport project.

Proposing Dynamic Optimization Strategy and Practice Method. Basing on the dynamic optimization method and dynamic optimization technique, from the pursuit of the best value of local top interface, to the pursuit of the overall design effect and the rationality and satisfaction of the comprehensive evaluation of gymnasiums of ice sport, systematical proposing the dynamic optimization strategy and practice method of the gymnasiums of ice sport design which possessing the micro foundation and operation.

\section{The Concrete Content of the Gymnasiums of Ice Sport Top Interface Design}

Under the dynamic characteristics framework which related the more broader interest groups and a full range of gymnasium of ice sport natural light environment, to take the dynamic design as an effective means of communicating between the light environment and the users behavior, making the 
more detailed analysis the problem between the complex natural light environment and the user behavior, which made a consensus among the stakeholders about the light environment optimization. Due to differences in the users demand, the functions obtained by the natural light environment is various and multi-level, it is necessary to make a mutual coordination and cooperation about the functions, according to the practical objective of the design project to focus to meet all the needs. Only by the objective master of the real needs of the gymnasium of ice sport users, it is possible for the architects to objectively and accurately accomplish the optimize design of the natural light environment at $\mathrm{t}$ gymnasiums of ice sport.

To Develop the Implementation Plan for the Gymnasiums of Ice Sport Top Interface Design Basing on the Dynamic Optimization of the Natural Light Environment. Through the collection and integration of the relevant design information about the gymnasiums of ice sport top interface and light environment present situation investigation on the part of cold areas in our country (Figure 2), making market analysis and locating position about the gymnasiums of ice sport top interface, formulating gymnasiums of ice sport top interface design implementation plan, to targeted and dynamically adapt the natural light to the specific needs of each energy-saving design scheme in the gymnasiums of ice sport top interface , in order to enhance the market competitiveness of the project design, and preparing for the "optimal "scheme selection and concrete.

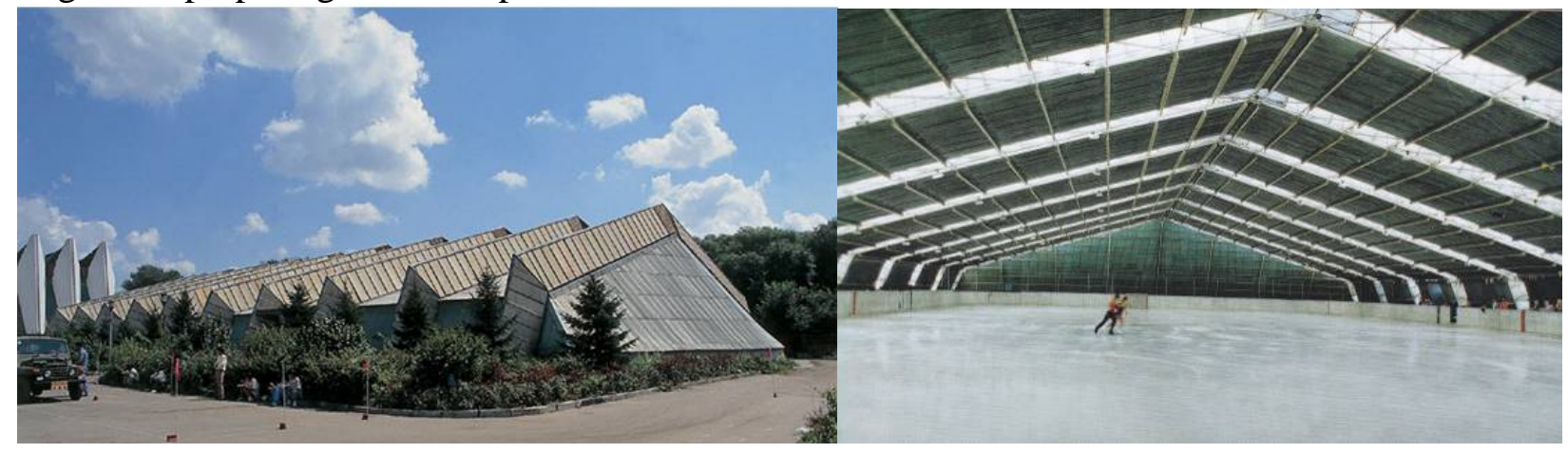

Fig.2 Jilin hockey training center

Constructing the Dynamic Optimization Model and the Dynamic Evaluation System of the Whole Life Cycle of the Gymnasiums of Ice Sport Top Interface. Making functional analysis of the gymnasiums of ice sport top interface, and explaining the functional requirements of the users of the gymnasiums of ice sport top interface. On the basis of qualitative and quantitative analysis of the function and cost, establishing the gymnasiums of ice sport top interface whole life cycle cost estimation model, to evaluate the function by using the relationship between the cost and function.

On the basis of constructing mathematical model about the competition hall internal visual comfort degree, taking the control variables for analysis and design, to minimize the energy consumption as the goal, establishing dynamic optimization model for the gymnasiums of ice sport top interface. And solving the optimal control variables of the dynamic model, making the technical indicators of the gymnasiums of ice sport top interface reached the "optimum", to explore the dynamic optimization technique of the gymnasiums of ice sport top interface, constructed whole life cycle dynamic evaluation system of the gymnasiums of ice sport top interface, to seek the lowest function cost.

Putting Forward the Dynamic Optimization Strategy for the Design of the Gymnasiums of Ice Sport Top Interface. Taking the dynamic optimization of the national light environment for the foundation, with the actual case launched the gymnasiums of ice sport top interface design scheme creation, through the identification of the advantages and disadvantages as well as on the performance of the project effect in the creation stage of the design scheme, to screen with the alternatives. And then carrying out the parametric model and dynamic natural light environment simulation, integrating the dynamic variables of the gymnasiums of ice sport top interface, through modifying the parametric statistics, the input and output and the model modification, to select the "optimal" scheme, which with a regional adaptability of the gymnasiums of ice sport top interface dynamic optimization. 
Finally, from the feedback of the operations, investment control and full life cycle dynamic evaluation of the "optimal" scheme, making a comprehensive summary about the dynamic optimization of the gymnasiums of ice sport top interface, to take the full life cycle cost advantage, and puts forward the dynamic optimization strategy and practice method to ensure the quality of the natural light environment of the gymnasiums of ice sport top interface design.

\section{Conclusions}

In order to realize the multifunctional utilization and the post game utilization of the gymnasium of ice sport, the natural lighting technology are introduced to the top interface design, it will increase the daytime sports hall utilization, thus saving the energy consumption for operation. In the face of the expectations and demands of different users, and different ice sports has a specific technical requirement about the natural light environment of the competition hall, the dynamic optimal design can be a link between them, to help their mutual communication and coordination. As renewable energy, natural light is not stable, with the different variables, such as the season, region, time, and so on, there are some changes. By the dynamic angle of view to establish dynamic model of natural light incidence, based on the whole life cycle optimizing the competition hall natural light environment, through the integrated optimization and the whole integration of high technology and low technology, active technology and passive technology in the various types of top interface design, optimize the appropriate technology that the "optimal" scheme can be derived from the gymnasium of ice sport top interface design which is based on the dynamic optimization of the natural light environment. Icing of the expectations and needs of different users, environmental behavioral methods can be a bond between them, to help them carry out communication and coordination. in a wider range of interest groups, and under the framework of the full range of the dynamic characteristics of natural light environment of gymnasium, use design as an effective means of communication between the environment and user behavior, to refine and analyze the problem of complex natural light environment and the user behavior, and to make consensus on light environment optimized between stakeholders. In the natural light environment design of gymnasium, it is necessary to give full consideration to the impact of various factors on the users' needs, to complete a full subjective analysis and subjective evaluation of the gymnasium natural light environment. Due to the differences of user demand, the behavioral function of natural light environment is multi-faceted and multi-level, in the design, these functions need to be coordinated according to the actual goal of the design project focused to meet. Only grasp of the real needs of the gymnasium users objectively, can the architects complete the optimization design of the gymnasium natural light environment objectively and accurately.

\section{Acknowledgement}

It is a project supported by Natural Science Foundation of Heilongjiang Province of China (E201242).

\section{References}

[1] Design code for sports building (JGJ 31-2003). Ministry of housing and urban-rural development of the people's republic of china. 2003: (10), 16(In Chinese)

[2] Li Binghua, Dong Qing. The construction of the International Design Consultants Limited group. Sports lighting design handbook. Beijing: China Electric Power Press, 2009: 286, 9(In Chinese)

[3] Robert.B.Stewart, Qiu Wanhua, The Method Basis of Value Engineering, Beijing: China Machine Press, (2007), p. 100-128(In Chinese)

[4] Liu Ying, Liu Deming. 2010, Design gymnasium natural light environment design based on value engineering. New building. No130 (3): 129-131. 